Vol. $13 \mathrm{~N}^{0}$ 2, pp. 99-113 December 1994

Universidad Católica del Norte

Antofagasta - Chile

\title{
UNIQUENESS OF BLOWING UP SOLUTIONS ON THE BOUNDARY IN QUASILINEAR ELLIPTIC EQUATIONS WITH NON HOMOGENEOUS TERM IN $L^{\infty}$
}

\author{
R.LETELIER* \\ Universidad de Concepción, Concepción, Chile \\ J.ORTEGA $A^{\dagger}$ \\ Universidad del Bio-Bio, Concepción, Chile
}

Abstract
We consider the quasilinear elliptic equation

$$
-\Delta u+H(x, u, D u)=f, \text { in } \Omega
$$

being $\Omega \subset I R^{N}, N \geq 1$, an open set with regular bounded boundary and $f \in E^{\infty}(\Omega)$. In this work we obtain upper estimates of the growth, near to the boundary, for any classic solution of $\left(E_{H}\right)$ and the exact behavior for any non negative solution of $\left(E_{H}\right)$ verifiying

$$
\lim _{\operatorname{dist}(x, \partial \Omega) \rightarrow 0} u(x)=+\infty .
$$

Finally, we prove the uniqueness of blow-up solutions of $\left(E_{H}\right)$.

Key words and phrases: Nonlinear elliptic equations, interior bounds, blow-up solutions, uniqueness.

*Partially supported by FONDECYT Grant $\mathrm{N}^{\circ} 92 / 0249$ and by DIUC Grant N० 911216-1.

${ }^{\dagger}$ Partially supported by DIPRODE U.B.B. Grant N $92 / 06061$. 


\section{Introduction}

In this work, we show upper estimates and uniqueness of unbounded solutions, in a sense that we will precise later, of the PDE in non divergent form

$$
-\Delta u+H(x, u, D u)=f, \text { in } \Omega
$$

being $\Omega$ an open set with bounded boundary of $I R^{N}, N \geq 1, f \in L^{\infty}(\Omega)$, $f \geq 0$, and $H: \Omega \times \overline{I R_{+}} \times I R^{N} \rightarrow I R_{+}$is an onto function verifying the following structural hypotheses (1):

i) $x \rightarrow H(x, r, q)$ belongs to $L^{\infty}(\Omega)$ and $(r, q) \rightarrow H(x, r, q)$ is continuous.

ii) $\left|H(x, r, q)-H\left(x, r, q^{\prime}\right)\right| \leq \mu \rho\left(\left|q-q^{\prime}\right|\right)$ almost everywhere on $\Omega, \forall r$, $\forall q, q^{\prime}$, for some $\mu \geq 0$, and $\rho: I R_{+} \rightarrow I R_{+}$is an onto increasing continuous function with $\rho\left(0^{+}\right)=0$.

iii) $H(x, r, q)-H(x, s, q) \geq \lambda[\beta(r)-\beta(s)], r \geq s$, almost every where on $\Omega, \forall r, \forall q, q^{\prime}$ $\lambda>0$ and $\beta: I R_{+} \rightarrow I R_{+}$is an onto increasing continuous function with $\beta\left(0^{+}\right)=0$.

\section{Remark 1}

Some particular choices of $H$ lead to the equations:

$$
\begin{gathered}
-\Delta u+\sum_{i=1}^{N} a_{i}(x) \frac{\partial u}{\partial x_{i}}+\beta(u)=f \\
-\Delta u+h(x, \nabla u)+\beta(u)=f
\end{gathered}
$$

where $k>0, \beta, h: I R_{+} \rightarrow I R_{+}$are continuous functions verifying $i$ ), ii), iii), and $a_{i}, f$ are locally bounded measurable functions on $\Omega$.

\section{Definition}

Let $1<s \leq \infty$. We say that $u \in W_{l o c}^{2, s}(\Omega)$ is a strong solution of $\left(E_{H}\right)$ if

$$
-\Delta u(x)+H(x, u(x), D u(x))=f \quad \text { a.e. } x \in \Omega .
$$

Our main results are,

i) Upper estimates of the growth, near the boundary, for any strong solution of $\left(E_{H}\right)$, particulary, these hold for every function $u$ which verifies $\left(E_{H}\right)$ on $\Omega$ and $u_{\mid a \Omega}$ bounded. 
ii) Existence of a unique behavior on the boundary, for any $u$, solution of the problem

$$
\begin{aligned}
-\Delta u+H(x, u, D u) & =f, \text { in } \Omega \\
\lim _{\operatorname{dist}(x, \partial \Omega) \rightarrow 0} u(x) & =+\infty .
\end{aligned}
$$

Therefore, a posteriori, the behavior on the boundary of the functions which verifies $\left(P_{H}\right)$, in strong sense, cannot be as general as

$$
\lim _{\operatorname{dist}(x, \partial \Omega) \rightarrow 0} u(x)=+\infty,
$$

but only one behavior, which is determined by the interior data of $\left(E_{H}\right)$.

iii) Uniqueness of strong solutions of $\left(P_{H}\right)$.

The following comparison result over strong solutions of $\left(E_{H}\right)$, will be very helpful.

Theorem 1 ([Di-Le1])

The structural hypotheses (1) implies,

If $u_{1}, u_{2} \in W_{l o c}^{2, \infty}(\Omega)$ verifies in strong sense

$$
-\Delta u_{1}+H\left(x, u_{1}, D u_{1}\right) \leq-\Delta u_{2}+H\left(x, u_{2}, D u_{2}\right), \text { in } \Omega,
$$

and

$$
\lim \sup \left(u_{1}-u_{2}\right)(x) \leq 0, \text { as } \operatorname{dist}(x, \partial \Omega) \rightarrow 0,
$$

then

$$
u_{1}(x) \leq u_{2}(x), x \in \Omega
$$

\section{Remark 2}

The previous theorem holds, in particular, when $u_{1}$ and/or $u_{2}$ are defined only in the interior, even if they are unbounded on $\partial \Omega$.

\section{Corollary 2 ([Di-Le1])}

The structural hypotheses (1) implies that:

if $u_{1}, u_{2} \in W_{\text {loc }}^{2, \infty}(\Omega)$, verifies, in strong sense,

$$
-\Delta u_{1}+H\left(x, u_{1}, D u_{1}\right)=-\Delta u_{2}+H\left(x, u_{2}, D u_{2}\right) \text {, in } \Omega
$$

and

$$
\lim \sup \left(u_{1}-u_{2}\right)(x)=0, \text { as } \operatorname{dist}(x, \partial \Omega) \rightarrow 0,
$$


then

$$
u_{1} \equiv u_{2} \text { in } \Omega \text {. }
$$

\section{Remark 3}

According to Corollary 2, the uniqueness of behavior on the boundary of bounded domains of unbounded strong solutions $u$, in the class $W_{l o c}^{2, \infty}(\Omega)$, of $\left(E_{H}\right)$, implies the uniqueness of unbounded solutions of $\left(E_{H}\right)$. See [Di-Le2].

\section{Remark 4}

About the existence of strong solutions of $\left(P_{H}\right)$. See [Le-Or].

The argumental work line of [Le-Or] is based on the results of the type LeraySchauder fixed point theorems (see [Gi- $\mathrm{Tr}_{\mathrm{r}}$ ], cap. 11), which need interior estimates of $u$ in $W_{l o c}^{1, \infty}(\Omega)$. In particular, the interior estimates of $u$ in $L_{l o c}^{\infty}(\Omega)$ are found through comparison arguments as the one indicated in Theorem 1 in [Di-Le1]. See also [Di-Le2]. In these papers the need to build adequate unbounded families of functions to compare them with the solutions of the problem $\left(P_{H}\right)$ are shown.

Taking into account the structure of $\left(E_{H}\right)$, a sufficient condition to obtain interior estimates of $u$ in $W_{l o c}^{1, \infty}(\Omega)$, see [Di-Le1], is the existence of an onto continuous function $\gamma: I R_{+} \rightarrow I R_{+}$, with $\gamma\left(0^{+}\right)=0$, verifying

$$
\int_{\tau}^{\infty} \frac{d s}{(\Gamma(s))^{\frac{1}{2}}}<\infty
$$

where,

$$
\Gamma(r)=\int_{0}^{r} \gamma(s) d s .
$$

Thus, through all this paper, we will suppose that $\left(H_{\gamma}\right)$ holds. Obviously the test functions will depend on $\gamma$. In particular, if $\gamma(r)=r^{m},\left(\mathcal{H}_{\gamma}\right)$ holds when $m>1$.

\section{Results}

To simplify this work, we only give the details for the following illustrative case,

Let $u \in W_{l o c}^{2, \infty}(\Omega)$ be a strong solution of the problem $(P)$,

$$
\begin{gathered}
-\Delta u(x)+|\nabla u(x)|^{k}+\lambda u^{m}(x)=f(x), x \in \Omega \\
\lim _{\operatorname{dist}(x, \partial \Omega) \rightarrow 0} u(x)=+\infty,
\end{gathered}
$$

where $\Omega$ is an open set with bounded boundary of $C^{2}$-class, $\lambda>0$, $m>1,0<k<2$ and $f \in L^{\infty}(\Omega), f \geq 0$. 
Lemma 3 ([Gi-Tr] $)$

Let $\Omega$ be a bounded domain of $I R^{N}, N \geq 1$, with non void $\partial \Omega$. Then if we write,

$$
d(x)=\operatorname{dist}(x, \partial \Omega), x \in I R^{N} .
$$

We have that,

$d(\cdot)$ is Lipschitz-continuous on $I R^{N}$. Moreover, if $\partial \Omega \in C^{k}, k \geq 2$, then there exists $\delta_{0}=\delta_{0}(\Omega)$ depending only on $\Omega$, such that,

$$
d(\cdot) \in C^{k}\left(B_{\delta_{0}}^{+}(\partial \Omega)\right)
$$

and

$$
|\nabla d(\cdot)| \equiv 1 \text {, en } B_{\delta_{0}}^{+}(\partial \Omega),
$$

where $B_{\delta_{0}}^{+}(\partial \Omega)=\left\{x \in \Omega, 0<d(x)<\delta_{0}\right\}$.

\section{Lemma 4}

If $\lambda>0, m>1$, then the following polynomials

$$
\begin{array}{ll}
P_{1}(r)=r\left[\lambda r^{m-1}-\left(\frac{2}{m-1}\right)\left(\frac{2}{m-1}+1\right)\right] & ; k>0 \\
P_{2}(r)=r\left[\lambda r^{m-1}-\left(\frac{2}{m-1}\right)\left(\frac{2}{m-1}+1\right)+\left(\frac{2}{m-1}\right)^{k} r^{k-1}\right] & ; k>1 \\
P_{3}(r)=r\left[\left(\frac{2-k}{k-1}\right)^{k} r^{k-1}-\left(\frac{2-k}{k-1}\right)\left(\frac{2-k}{k-1}+1\right)\right] & ; 1<k<2
\end{array}
$$

have a unique positive root.

Proof:

Let $P(r) \in\left\{P_{1}(r), P_{2}(r), P_{3}(r)\right\}$. Then $P(r)$ verifies

1. $P(0)=0$

2. $P^{\prime}(0)<0$

3. $P^{\prime \prime}(r)>0, \forall r>0$, (i.e. $P(r)$ is strictly convex).

From here, it follows that each polynomial $P(r)$ has a unique positive root. Furthermore, in the cases of $P_{1}$ and $P_{3}$ their roots can be explicitly calculated, obtaining

$$
c_{1}=\left[\frac{1}{\lambda}\left(\frac{2}{m-1}\right)\left(\frac{2}{m-1}+1\right)\right]^{\frac{1}{m-1}}
$$

this the unique positive root of $P_{1}$, and

$$
c_{3}=\frac{(k-1)}{(2-k)}^{-\frac{(2-k)}{(k-1)}}
$$


is the unique positive root of $P_{3}$

\section{Remark 5}

It is easy to prove the inequalities

$$
\forall r \geq 0: P_{1}(r) \leq P_{2}(r) \text { and } P_{3}(r) \leq P_{2}(r)
$$

therefore,

$$
c_{2} \leq c_{1} \text { and } c_{2} \leq c_{3}
$$

\section{Theorem 5 (upper estimate of any solution of $(E)$ )}

Let $\Omega$ be an open set with bounded boundary of $C^{2}$-class and $f \in L^{\infty}(\Omega), f \geq 0$. Assume that $\lambda>0, m>1,0<k<2$ and $c_{1}, c_{2}, c_{3}$ the roots obtained in Lemma 4 .

If $u \in W_{l o c}^{2, \infty}(\Omega)$ is a strong solution of the problem $(P)$, then

$$
\lim _{\operatorname{dist}(x, \partial \Omega) \rightarrow 0} \sup \frac{u(x)}{w(x)} \leq C,
$$

where $w=w(\operatorname{dist}(x, \partial \Omega), k, m, \lambda, f)$ is a known function which is obtained explicitly and $C=\max \left\{c_{1}, c_{2}, c_{3}\right\}$.

In particular, we have the following estimates,

$$
\begin{aligned}
& \text { 1.- } 0<k<\frac{2 m}{m+1} \Rightarrow \lim _{\text {dist }(x, \partial \Omega) \rightarrow 0} \sup u(x) d(x)^{\frac{2}{m-1}} \leq c_{1} \\
& \text { 2.- } k=\frac{2 m}{m+1} \quad \Rightarrow \quad \lim _{\text {dist }(x, \partial \Omega) \rightarrow 0} \sup u(x) d(x)^{\frac{2}{m-1}} \leq c_{2} \\
& \text { 3.- } \frac{2 m}{m+1}<k<2 \Rightarrow \lim _{\text {dist }(x, \partial \Omega) \rightarrow 0} \sup u(x) d(x)^{\frac{2-k}{k-1}} \leq c_{3} .
\end{aligned}
$$

Proof:

Let $\delta_{0}>0$ be the constant given in Lemma $\left.3, \delta \in\right] 0, \delta_{0}[$ and $\epsilon>0$.

Now, we define the function

$$
W(x)=W_{\epsilon, \delta}(x)=\left(c_{i}+\epsilon\right)[d(x)-\delta]^{-\alpha}+c_{\epsilon}, x \in \Omega_{-\delta},
$$

where $\alpha \in\left\{\left(\frac{2}{m-1}\right),\left(\frac{2-k}{k-1}\right)\right\}, c_{i}$ is the positive root of $P_{i}, c_{\epsilon}>0$ is a constant to be determined, and

$$
\Omega_{-\delta}=\{x \in \Omega: \operatorname{dist}(x, \partial \Omega)>\delta\}, \Gamma_{\delta}=\Omega_{\delta} \backslash \Omega_{-\delta} .
$$


To simplify, we write $\beta(x)=d(x)-\delta$. Thus through direct calculation over the function $W \in C^{2}\left(\Omega_{-\delta}\right)$, we obtain the equality,

$$
\begin{gathered}
-\Delta W(x)+|\nabla W(x)|^{k}+\lambda W^{m}(x)-f(x)= \\
-\alpha(\alpha+1)\left(c_{i}+\epsilon\right) \beta^{-(\alpha+2)}|\nabla d|^{2}+\alpha\left(c_{i}+\epsilon\right) \beta^{-(\alpha+1)} \Delta d \\
+\alpha^{k}\left(c_{i}+\epsilon\right)^{k} \beta^{-(\alpha+1) k}|\nabla d|^{k}+\lambda\left[\left(c_{i}+\epsilon\right) \beta^{-\alpha}+c_{\epsilon}\right]^{m}-f
\end{gathered}
$$

Since for $x \in \Omega_{-\delta} \cap \Gamma_{\delta_{0}},|\nabla d(x)|=1$ holds, then,

$$
\begin{gathered}
-\Delta W(x)+|\nabla W(x)|^{k}+\lambda W^{m}(x)-f(x) \geq \\
-\alpha(\alpha+1)\left(c_{i}+\epsilon\right) \beta^{-(\alpha+2)}-\alpha\left(c_{i}+\epsilon\right) \beta^{-(\alpha+1)}\|\Delta d\|_{L^{\infty}(\Omega)} \\
+\alpha^{k}\left(c_{i}+\epsilon\right)^{k} \beta^{-(\alpha+1) k}+\lambda\left(c_{i}+\epsilon\right)^{m} \beta^{-\alpha m}-\|f\|_{L^{\infty}(\Omega)}, x \in \Omega_{-\delta} \cap \Gamma_{\delta_{0}} .
\end{gathered}
$$

Let us analyze each case which appears in the theorem.

\section{Case 1}

Let

$$
0<k<\frac{2 m}{m+1}
$$

then choosing $\alpha=\frac{2}{m-1}$, we obtain

$$
\alpha m=\alpha+2>(\alpha+1) k
$$

and therefore,from $(i)$, we can write, in $\Omega_{-\delta} \cap \Gamma_{\delta_{0}}$

$$
\begin{gathered}
-\Delta W(x)+|\nabla W(x)|^{k}+\lambda W^{m}(x)-f(x) \geq \\
\beta^{-(\alpha+2)}\left[\lambda\left(c_{1}+\epsilon\right)^{m}-\alpha(\alpha+1)\left(c_{1}+\epsilon\right)-\alpha\left(c_{1}+\epsilon\right) \beta\|\Delta d\|_{L^{\infty}(\Omega)}\right. \\
\left.+\alpha^{k}\left(c_{1}+\epsilon\right)^{k} \beta^{(\alpha+2)-(\alpha+1) k}-\|f\|_{L^{\infty}(\Omega)} \beta^{(\alpha+2)}\right] .
\end{gathered}
$$

Since $c_{1}$ is the positive root of $P_{1}$, for $\epsilon>0$ small enough, we have the inequality

$$
\lambda\left(c_{1}+\epsilon\right)^{m}-\alpha(\alpha+1)\left(c_{1}+\epsilon\right)>0,
$$

and then, $\forall \epsilon>0$, there exists $\left.\delta_{1}=\delta_{1}(\lambda, \epsilon) \in\right] 0, \delta_{0}[$, such that,

$$
\begin{gathered}
{\left[\lambda\left(c_{1}+\epsilon\right)^{m}-\alpha(\alpha+1)\left(c_{1}+\epsilon\right)-\alpha\left(c_{1}+\epsilon\right) \delta_{1}\|\Delta d\|_{L^{\infty}(\Omega)}+\right.} \\
\left.\alpha^{k}\left(c_{1}+\epsilon\right)^{k} \delta_{1}^{(\alpha+2)-(\alpha+1) k}-\|f\|_{L^{\infty}(\Omega)} \delta_{1}^{(\alpha+2)}\right]>0 .
\end{gathered}
$$


Thus if we choose $\delta \in] 0, \delta_{1 / 2}[$, such that

$$
0<\delta<d(x)<\delta_{1}<\delta_{0}
$$

then we have that

$$
0<\beta(x)=d(x)-\delta<\delta_{1},
$$

and therefore, from (ii),

$$
\begin{gathered}
-\Delta W(x)+|\nabla W(x)|^{k}+\lambda W^{m}(x)-f(x) \geq \\
\beta^{-(\alpha+2)}\left[\lambda\left(c_{1}+\epsilon\right)^{m}-\alpha(\alpha+1)\left(c_{1}+\epsilon\right)-\alpha\left(c_{1}+\epsilon\right) \beta\|\Delta d\|_{L^{\infty}(\Omega)}\right. \\
\left.+\alpha^{k}\left(c_{1}+\epsilon\right)^{k} \beta^{(\alpha+2)-(\alpha+1) k}-\|f\|_{L^{\infty}(\Omega)} \beta^{(\alpha+2)}\right]>0, \text { in } \Omega_{-\delta} \cap \Gamma_{\delta_{1}} .
\end{gathered}
$$

Let

$$
c_{\epsilon}=\max \left\{u(x): d(x) \geq \delta_{1}\right\}
$$

then

$$
\lim _{d(x) \rightarrow \delta} \sup [W-u](x)=+\infty,
$$

and

$$
W(x) \geq u(x), x \in \partial \Omega_{-\delta_{1}} .
$$

Finally, from Theorem 1 we obtain,

$$
u(x) \leq\left(c_{1}+\epsilon\right)[d(x)-\delta]^{\frac{-2}{m-1}}+\max \left\{u(x): d(x) \geq \delta_{1}\right\}, x \in \Omega_{-\delta},
$$

therefore,

$$
u(x)[d(x)-\delta]^{\frac{2}{m^{-1}}} \leq\left(c_{1}+\epsilon\right)+c_{\epsilon}[d(x)-\delta]^{\frac{2}{m-1}}, x \in \Omega_{-\delta} .
$$

Firstly, taking $\delta \rightarrow 0$ and then making $d(x) \rightarrow 0$, in the previous inequality we have that $\forall \epsilon>0$ :

$$
\lim _{\operatorname{dist}(x, \partial \Omega) \rightarrow 0} \sup u(x) d(x)^{\frac{2}{m-1}} \leq\left(c_{1}+\epsilon\right),
$$

therefore, we conclude that

$$
\lim _{\operatorname{dist}(x, \partial \Omega) \rightarrow 0} \sup u(x) d(x)^{\frac{\partial}{m-1}} \leq c_{1} .
$$

For the other cases, we proceed in a similar way obtaining the following results.

\section{Case 2}

If $k=\frac{2 m}{m+1}$, then for $\alpha=\frac{2}{m-1}$, we have

$$
\alpha m=\alpha+2=(\alpha+1) k
$$


and furthermore

$$
\lim _{\operatorname{dist}(x, \partial \Omega) \rightarrow 0} \sup u(x) d(x)^{\frac{2}{m-1}} \leq c_{2} .
$$

Note that when the structure of the equation is given for this choice of $k$, the polynomial $P_{2}(r)$ can be written as,

$$
\begin{aligned}
P_{2}(r) & =r\left[\lambda r^{m-1}-\left(\frac{2}{m-1}\right)\left(\frac{2}{m-1}+1\right)+\left(\frac{2}{m-1}\right)^{\frac{2 m}{m+1}} r^{\frac{m-1}{m+1}}\right] \\
& =r\left[\lambda r^{\frac{2 k-2}{2-k}}-\left(\frac{2-k}{k-1}\right)\left(\frac{2-k}{k-1}+1\right)+\left(\frac{2-k}{k-1}\right)^{k} r^{k-1}\right] .
\end{aligned}
$$

\section{Case 3}

If $\frac{2 m}{m+1}<k<2$, then for $\alpha=\frac{2-k}{k-1}$, we have that

$$
\alpha m<\alpha+2=(\alpha+1) k \text {. }
$$

Furthermore

$$
\lim _{\operatorname{dist}(x, \partial \Omega) \rightarrow 0} \sup u(x) d(x)^{\frac{2-k}{k-1}} \leq c_{3}
$$

\section{Remark 6}

The upper estimates obtained in Theorem 5 allow us to show, in particular, results of non existence of strong solutions of

$$
\begin{aligned}
-\Delta u+H(x, u, D u) & =f \quad, \text { in } \Omega \\
\lim _{\operatorname{dist}(x . \partial \Omega) \rightarrow 0} \frac{u(x)}{\varphi(x)} & =1
\end{aligned}
$$

assuming that $\varphi \epsilon C(\Omega)$ is unbounded, and increases faster than the upper estimate obtained in Theorem 5 , on the boundary.

Lemma 6 [Gi-Tr], [Lieb].

Let $\Omega \subseteq I R^{n}$ be an open set with bounded boundary of $C^{2}$-class. For $\delta>0$, we define the following sets,

$$
\Omega_{\delta}=\left\{x \in I R^{n} / \operatorname{dist}(x, \bar{\Omega})<\delta\right\} \text { and } \Gamma_{\delta}=\Omega_{\delta} \backslash \Omega_{-\delta}
$$

where $\Omega_{-\delta}$ was defined in the proof of Theorem 5 . Therefore, we can write $\Omega_{0}=\Omega$.

We have that, there exists a function $d \epsilon C^{k}\left(I R^{n}, I R\right)$, such that 
i) $\quad d(x)=\operatorname{dist}(x, \partial \Omega) \quad, x \in B_{\delta_{0}}^{+}(\partial \Omega)$

ii) $\quad d(x)=-\operatorname{dist}(x, \partial \Omega) \quad, x \in \Omega_{\delta_{0}} \backslash \Omega$

iii) $d(x) \geq \delta_{0} \quad, x \in \Omega_{-\delta_{0}}$

iv) $\quad d(x) \leq-\delta_{0} \quad, x \in I R^{n} \backslash \Omega_{\delta_{0}}$.

Note that i) and ii) implies that

$$
|\nabla d(x)|=1, \text { when } x \in \Gamma_{\delta_{0}} .
$$

\section{Remark 7}

Recall that, from Lemma 3 it is known that if $\Omega \subseteq I R^{n}$ is an open set with bounded boundary of $C^{k}$-class, then there exists a constant $\delta_{0}>0$ such that $\operatorname{dist}(x, \partial \Omega)$ is of $C^{k}$-class on $\Omega-\Omega_{-\delta_{0}}$.

Theorem 7 (Growth rate of the unbounded solutions of $(E)$ )

Let $\Omega \subseteq I R^{n}$ be an open set with bounded boundary of $C^{2}$-class, $\lambda>0$, $m>1,0<k<2$ and $f \in L^{\infty}(\Omega), f \geq 0$.

If $u \in W_{\text {loc }}^{2, \infty}(\Omega)$ is a strong solution of $(P)$, then the growth rate of $u$ is,

$$
\lim _{\operatorname{dist}(x, \partial \Omega) \rightarrow 0} u(x) d(x)^{\frac{2}{m-1}}=C,
$$

where $C=C(\lambda, m, f, k) \in I R^{+}$is a known constant and $\alpha_{i} \in\left\{\frac{2}{m-1}, \frac{2-k}{k-1}\right\}$.

In particular, we prove that if $c_{i}$ is the positive root of $P_{i}$, given in Lemma 4, then

1.- $\quad 0<k<\frac{2 m}{m+1} \Rightarrow \lim _{\text {dist }(x, \partial \Omega) \rightarrow 0} u(x) d(x)^{\frac{2}{m-1}}=c_{1}$

2.- $\quad k=\frac{2 m}{m+1} \Rightarrow \lim _{\operatorname{dist}(x, \partial \Omega) \rightarrow 0} u(x) d(x)^{\frac{2}{m-1}}=c_{2}$

3.- $\frac{2 m}{m+1}<k<2 \Rightarrow \lim _{\text {dist }(x, \partial \Omega) \rightarrow 0} u(x) d(x)^{\frac{2-k}{k-1}}=c_{3}$.

Proof:

Let $\delta_{0}>0$ be the constant given in Lemma 3. For $\left.\delta \in\right] 0, \delta_{0}[$ and $\epsilon \in] 0, c_{i}[$, fixed but arbitrary, we define, in $\Omega_{\delta}$, the function

$$
w(x)=w_{\epsilon, \delta}(x)=\left(c_{i}-\epsilon\right)[d(x)+\delta]^{-\delta}-c_{\epsilon}, \forall x \in \Omega_{\delta},
$$

where $\alpha \in\left\{\frac{2}{m-1}, \frac{2-k}{k-1}\right\}, c_{i}$ is the positive root of the polynomial $P_{i}$ and $c_{\epsilon}>0$ is a constant to determine.

We write $\beta=[d+\delta]$ and via direct calculations over the function $w \in C^{2}\left(\Omega_{\delta}\right)$, we obtain

$$
-\Delta w(x)+|\nabla w(x)|^{k}+\lambda w^{m}(x)-f(x)=-\alpha(\alpha+1)\left(c_{i}-\epsilon\right) \beta^{-(\alpha+2)}|\nabla d|^{2}+
$$


$\alpha\left(c_{i}-\epsilon\right) \beta^{-(\alpha+1)} \Delta d+\alpha^{k}\left(c_{i}-\epsilon\right)^{k} \beta^{-(\alpha+1) k}|\nabla d|^{k}+\lambda\left[\left(c_{i}-\epsilon\right) \beta^{-\alpha}-c_{\epsilon}\right]^{m}-f$, in $\Omega$.

Since in the region $\Omega \cap \Gamma_{\delta_{0}}$, we have that $|\nabla d(x)|=1$, then

$$
\begin{gathered}
-\Delta w(x)+|\nabla w(x)|^{k}+\lambda w^{m}(x)-f(x) \leq \\
-\alpha(\alpha+1)\left(c_{i}-\epsilon\right) \beta^{-(\alpha+2)}+\alpha\left(c_{i}-\epsilon\right) \beta^{-(\alpha+1)}\|\Delta d\|_{L^{\infty}(\Omega)} \\
+\alpha^{k}\left(c_{i}-\epsilon\right)^{k} \beta^{-(\alpha+1) k}+\lambda\left[\left(c_{i}-\epsilon\right) \beta^{-\alpha}-c_{\epsilon}\right]^{m}-\|f\|_{L^{\infty}(\Omega)}, \text { in } \Omega \cap \Gamma_{\delta_{0}} .
\end{gathered}
$$

Now, let us analyze each case given in the theorem.

\section{Case 1}

Assume that $0<k<\frac{2 m}{m+1}$, therefore choosing,

$$
c_{\varepsilon}=\left(c_{1}-\epsilon\right)(2 \delta)^{-\delta} \text { and } \alpha=\frac{2}{m-1}
$$

we obtain

$$
\alpha m=\alpha+2>(\alpha+1) k
$$

for every $x \in \Omega \cap \Gamma_{\delta}$, we have

$$
0<\left(c_{1}-\epsilon\right) \beta(x)^{-\alpha}-c_{\varepsilon} .
$$

Thus,

$$
\left[\left(c_{1}-\epsilon\right) \beta^{-\alpha}-c_{\varepsilon}\right]^{m} \leq\left[\left(c_{1}-\epsilon\right) \beta^{-\alpha}\right]^{m} \quad x \in \Omega \cap \Gamma_{\delta} .
$$

By using the previous inequality in $\Omega \cap \Gamma_{\delta}$, we have that,

$$
\begin{gathered}
-\Delta w(x)+|\nabla w(x)|^{k}+\lambda w^{m}(x)-f(x) \leq \\
\beta^{-(\alpha+2)}\left[\lambda\left(c_{1}-\epsilon\right)^{m}-\alpha(\alpha+1)\left(c_{1}-\epsilon\right)+\alpha\left(c_{1}-\epsilon\right) \beta\|\Delta d\|_{L^{\infty}(\Omega)}\right. \\
\left.+\alpha^{k}\left(c_{1}-\epsilon\right)^{k} \beta^{(\alpha+2)-(\alpha+1) k}+\|f\|_{L^{\infty}(\Omega)} \beta^{(\alpha+2)}\right], \quad x \in \Omega \cap \Gamma_{\delta} .
\end{gathered}
$$

On the other hand, since $c_{1}$ is the unique positive root of the polynomial $P_{1}$, and $P_{1}$ tends to $\infty$ as $r \rightarrow \infty$, we have that for $\epsilon>0$, small enough, but arbitrary,

$$
\lambda\left(c_{1}-\epsilon\right)^{m}-\alpha(\alpha+1)\left(c_{1}-\epsilon\right)<0
$$

and then, there exists $\left.\delta_{1}=\delta_{1}(\lambda, \varepsilon) \in\right] 0, \delta_{0}[$, such that,

$$
\begin{gathered}
{\left[\lambda\left(c_{1}-\epsilon\right)^{m}-\alpha(\alpha+1)\left(c_{1}-\epsilon\right)+\alpha\left(c_{1}-\epsilon\right) \delta_{1}\|\Delta d\|_{L^{\infty}(\Omega)}+\right.} \\
\left.+\alpha^{k}\left(c_{1}-\epsilon\right)^{k} \delta_{1}^{(\alpha+2)-(\alpha+1) k}+\|f\|_{L \infty(\Omega)} \delta_{1}^{(\alpha+2)}\right]<0
\end{gathered}
$$

Therefore, if $\delta \in] o, \delta_{1 / 2}[$, then

$$
0<d(x)<\delta
$$


and

$$
0<\beta(x)=d(x)+\delta<2 \delta<\delta_{1},
$$

consequently from (ii) and (iii), if $x \in \Omega \cap \Gamma_{\delta}$ we have that,

$$
\begin{gathered}
-\Delta w(x)+|\nabla w(x)|^{k}+\lambda w^{m}(x)-f(x) \leq \\
\beta^{-(\alpha+2)}\left[\lambda\left(c_{1}-\epsilon\right)^{m}-\alpha(\alpha+1)\left(c_{1}-\epsilon\right)+\alpha\left(c_{1}-\epsilon\right) \beta\|\Delta d\|_{L^{\infty}(\Omega)}\right. \\
\left.+\alpha^{k}\left(c_{1}-\epsilon\right)^{k} \beta^{(\alpha+2)-(\alpha+1) k}+\|f\|_{L^{\infty}(\Omega)} \beta^{(\alpha+2)}\right] \leq 0, \quad x \in \Omega \cap \Gamma_{\delta} .
\end{gathered}
$$

Since $w$ verifies,

$$
\lim _{\operatorname{dist}(x, \partial \Omega) \rightarrow 0}[w-u](x)=-\infty
$$

and

$$
w(x) \leq u(x), \quad \text { on } \partial \Omega_{-\delta},
$$

theorem 1 allows us to conclude that, for every $x \in \Omega \cap \Gamma_{\delta}$

$$
\left(c_{1}-\epsilon\right)[d(x)+\delta]^{\frac{-2}{m-1}}-\left(c_{1}-\epsilon\right)[2 \delta]^{\frac{-2}{m-1}} \leq u(x) .
$$

On the other hand, since $2 \delta<\delta_{1}$, then

$$
\left(c_{1}-\epsilon\right)[d(x)+\delta]^{\frac{-2}{m-1}}-\left(c_{1}-\epsilon\right)\left[\delta_{1}\right]^{\frac{-2}{m-1}} \leq u(x), x \in \Omega \cap \Gamma_{\delta}
$$

that is,

$$
\left(c_{1}-\epsilon\right)-\left(c_{1}-\epsilon\right)\left[\delta_{1}\right]^{\frac{-2}{m-1}}[d(x)+\delta]^{\frac{2}{m-1}} \leq u(x)[d(x)+\delta]^{\frac{2}{m-1}}, x \in \Omega \cap \Gamma_{\delta} .
$$

Now, taking limit $\delta \rightarrow 0$ in $(i v)$, and then making $d(x) \rightarrow 0$, for $\epsilon>0$, small enough, we have that

$$
\lim _{\operatorname{dist}(x, \partial \Omega) \rightarrow 0} \inf u(x) d(x)^{\frac{2}{m-1}} \geq\left(c_{1}-\epsilon\right),
$$

hence,

$$
\lim _{\operatorname{dist}(x, \partial \Omega) \rightarrow 0} \inf u(x) d(x)^{\frac{3}{m-1}} \geq c_{1} .
$$

Finally, from $(v)$ and Theorem 5 , it is immediately that

$$
\lim _{\operatorname{dist}(x, \partial \Omega) \rightarrow 0} u(x) d(x)^{\frac{2}{m-1}}=c_{1} .
$$

In the same way, we obtain the following results,

\section{Case 2}

Let $k=\frac{2 m}{m+1}$ then for $\alpha=\frac{2}{m-1}$. 
We have that

$$
\alpha m=\alpha+2=(\alpha+1) k,
$$

and

$$
\lim \inf _{\operatorname{dist}(x, \partial \Omega) \rightarrow 0} u(x) d(x)^{\frac{2}{m-1}} \geq c_{2} .
$$

\section{Case 3}

Let $\frac{2 m}{m+1}<k<1$ then for $\alpha=\frac{2-k}{k-1}$.

We have that,

$$
\alpha m<\alpha+2=(\alpha+1) k
$$

and

$$
\lim \inf _{\operatorname{dist}(x, \partial \Omega) \rightarrow 0} u(x) d(x)^{\frac{2-k}{k-1}} \geq c_{3} .
$$

These results and Theorem 5 conclude the proof of the theorem.

\section{Remark 8}

In particular, the previous theorem shows a non-existence result of strong solutions for the problem

$$
\begin{aligned}
-\Delta u+H(x, u, D u) & =f, \text { in } \Omega \\
\lim _{\text {dist }(x, \partial \Omega) \rightarrow 0} \frac{u(x)}{\varphi(x)} & =1,
\end{aligned}
$$

assuming that $\varphi$ is a unbounded continuous function on $\Omega$ and for some $q>0$ :

$$
\lim _{\operatorname{dist}(x, \partial \Omega) \rightarrow 0} \varphi(x) \operatorname{dist}(x, \partial \Omega)^{-q} \notin\left\{c_{1}, c_{2}, c_{3}\right\} .
$$

Theorem 8 (Uniqueness of the unbounded solutions of the equation $(E))$

If $\Omega \subseteq I R^{n}$ is an open set with bounded boundary of $C^{2}$-class, $\lambda>0, m>1,0<k<2$ and $f \in L^{\infty}(\Omega), f \geq 0$, then the problem $(P)$ has, at most, a unique strong solution in the class $W_{l o c}^{2, \infty}(\Omega)$. 
Proof:

Let $u_{1}, u_{2} \in W_{l o c}^{2, \infty}(\Omega)$ be two strong solutions of the problem $(P)$. Then from Theorem 4, we have that,

$$
\lim _{\operatorname{dist}(x, \partial \Omega) \rightarrow 0} \frac{u_{1}(x)}{u_{2}(x)}=\lim _{\operatorname{dist}(x, \partial \Omega) \rightarrow 0} \frac{u_{2}(x)}{u_{1}(x)}=1,
$$

therefore

$$
\lim _{\operatorname{dist}(x, \partial \Omega) \rightarrow 0}\left(u_{1}-u_{2}\right)(x)=0
$$

since it is also true

$$
\begin{gathered}
-\Delta u_{1}(x)+\left|\nabla u_{1}(x)\right|^{k}+\lambda u_{1}^{m}(x)-f(x)= \\
-\Delta u_{2}(x)+\left|\nabla u_{2}(x)\right|^{k}+\lambda u_{2}^{m}(x)-f(x), \quad x \in \Omega,
\end{gathered}
$$

the comparison results allow us to obtain,

$$
u_{1}(x)=u_{2}(x), x \in \Omega,
$$

and this completes the proof.

\section{Bibliography}

[Di-Le1] G.Díaz, R.Letelier. Local estimates: uniqueness of solutions of some nonlinear elliptic equations. Revista de la Real Academia de Ciencias de Madrid. To appear.

[Di-Le2] G.Diaz, R.Letelier. Explosive solutions in quasilinear elliptic equations: Existence and uniqueness. Nonlinear Analysis, Theory Meth. and Appl., Vol. 20, No 2, pp 97-125, 1993.

[Gi-Tr] D.Girbarg, N.Trudinger. Elliptic Partial Differential Equations of Second Order . Springer Verlag, 1983.

[L-L] J.M.Lasry, P.L.Lions. Non linear elliptic equations with singular boundary conditions and stochastic control with state constraints. Math Ann. 283, 1989 , pg. 583-630.

[Le-Or] R.Letelier, J.Ortega. Local gradient estimates and existence of minimal solutions of some semilinear elliptic equations blowing up on the boundary. Revista de la Academia Canaria de Ciencias, Vol. V, 1994. To appear.

[Lieb] Lieberman G.M.. Regularized distance and its applications. Pacific J. Math. 117,1985, pg. 336-353. 
Received: 02 May, 1994

R.Letelier

Departamento de Métodos Matemáticos

Universidad de Concepción

Casilla 4009. Concepción. Chile.

J.Ortega

Departamento de Materrática

Universidad del Bío-Bío

Casilla 5-C. Concepción. Chile. 